\title{
Identification and Estimation of Gaussian Affine Term Structure Models with Regime Switching
}

\author{
Gang Wang1,2 \\ ${ }^{1}$ School of Finance, Shanghai University of Finance and Economics, Shanghai, China \\ ${ }^{2}$ Shanghai Key Laboratory of Financial Information Technology, Shanghai, China \\ Email: delta9527@gmail.com
}

Received 16 February 2014; revised 19 March 2014; accepted 3 April 2014

Copyright (C 2014 by author and Scientific Research Publishing Inc.

This work is licensed under the Creative Commons Attribution International License (CC BY). http://creativecommons.org/licenses/by/4.0/

c) (i) Open Access

\section{Abstract}

We establish that [1]'s parameters are universally unidentified and a subset of their parameterization is over identified. As a solution to the problem with the identifiability, we propose a new representation of double-regime three-factor GDTSMs whose parameters are just-identified when the number of the pricing-with-error yields equals 2 . This new parametrization has another advantage over $[2]$ in that we can back out $\mathbb{Q}$ parameters and $\mathbb{P}$ parameters separately and make the estimation of structural parameters easier. Finally, we show that regime-switching three-factor arbitrage-free dynamic Nelson-Siegel model is a restricted special case of our model.

\section{Keywords}

\section{Regime Switching, GDTSMs, Identification, Estimation}

\section{Introduction}

After [2] proposed the single factor Gaussian affine term structure model. The class of Gaussian affine term structure models (GDTSMs) has been generalized and developed by, [3] [4], and [5] and has become the basic workhorse in macroeconomics and finance for purposes of using a no-arbitrage framework for studying the relations between yields on assets of different maturities. [4] and [5] find the Gaussian form of three-factor affine term structure model describes US treasury yields better than other forms. However, there is an extensive empirical literature on bond yields (particularly short-term rates) that suggests that "switching-regime" models describe the historical interest rate data better than single-regime models (see, for example, [1] [6] and [7]).

[1] develop a discrete-time multi-factor DTSM with the following features: 1) within each regime the 
short-term interest rate follows a three-factor Gaussian model with state-dependent market prices of factor risks; 2) there are two regimes characterized by low $(\mathrm{L})$ and high $(\mathrm{H})$ volatility, and the transitions between these regimes under the historical measure $\mathrm{P}$ are governed by a Markov process with regime-shift probabilities $\pi_{t}^{\mathbb{P}^{i j}}(i, j=H, L)$ that depend on the risk factors underlying changes in the shape of the yield curve; and 3) regime-shift risks are priced. This model yields exact closed-form solutions for bond prices, and an analytic representation of the likelihood function that they use in their empirical analysis of US. Treasury zero-coupon bond yields. Expected excess returns are decomposed into two components, which are associated with regime-shift and factor risks, respectively.

But in the practical experience of those who have used DSY model are tremendous numerical challenges in estimating the necessary parameters from the data due to highly non-linear and badly behaved likelihood surfaces. For example, [1] reported:

... Even with these normalizations/constraints, the resulting maximally flexible $A_{0}^{R S}(3)$ model (with restrictions for analytical pricing) involves a high dimensional parameter space...

Another problem with DSY model is its identification. We find that DSY model parameters are universally unidentified. If there are some parameters in the model that are unidentified, then it will be wrong to make conclusions from its parameters' estimate, let us say about how regime-shift risks are priced.

This paper proposes solution to them and other problems with regime-switching affine term structure model of [1] based on what we will refer to as their reduced-form representation. For a popular class of regime-switching Gaussian affine term structure models-namely, those for which the model is claimed to price exactly a subset of $N$ linear combinations of observed yields, where $N$ is the number of unobserved pricing factors - this reduced form is a restricted regime-switching multivariate linear regression in the observed set of yields.

One implication is that the parameters of these reduced-form representations contain all the observable implications of [1] regime-switching Gaussian affine term structure model for the sample of observed data, and can therefore be used as a basis for assessing identification. If more than one value for the parameter vector of interest is associated with the same reduced-form parameter vector, then the model is unidentified at that point and there is no way to use the observed data to distinguish between the alternative possibilities. [8] has applied this idea to affine term structure models with single regime. In this paper, we use it to demonstrate that [1] is in fact unidentified, an observation that our paper is the first to point out. This issue of identification is one factor that contributes to the numerical difficulties for conventional methods.

A second and completely separate contribution of the paper is that we propose our canonical representation of GDTSMs, which is then used in double-regime environment as a new form of regime-switching GDTSMs. Using this form of representation, it is possible for the parameters of interest to be inferred directly from estimates of the reduced-form parameters themselves. This is a very useful result because the latter are often simple regime-switching OLS coefficients. Although translating from reduced-form parameters into structural parameters involves a mix of analytical and numerical calculations, the numerical component is far simpler than that associated with the usual approach of trying to find the maximum of the likelihood surface directly as a function of the structural parameters.

There have been several other recent efforts to use new development in GDTSMs for multi-regime consideration. [9] developed a no-arbitrage representation of a dynamic Nelson-Siegel model of interest rates that gives a convenient representation of level, slope and curvature factors. For example, [10] presents an affine, arbitrage-free, regime-switching dynamic Nelson-Siegel model of the term structure (Regime-Switching AFNS). We show that it is a special case of our new form of regime-switching GDTSMs.

The chief difference between this paper and other relevant papers is that they focus on how the regime-switching GDTSMs should be represented, whereas we also examine how the parameters of the regimeswitching GDTSMs are to be estimated.

The rest of the paper is organized as follows. Section 2 describes [1] regime-switching Gaussian affine term structure model. Section 3 investigates the mapping from structural to reduced-form parameters. We establish that the canonical forms of [1] are universally unidentified and a subset of their parameterization is over identified. In Section 4, we propose a new representation. We establish when this representation is just-identified and how the parameters are to be estimated. In Section 5, we examine Regime-Switching AFNS's representation. We establish that it is the constrained special case of our representation. Section 6 concludes. 


\section{Regime-Switching Gaussian Affine Term Structure Model}

In this section, we just briefly describe the model set by [1]. Given the time $t+1$ regime $S_{t+1}=j$, under the risk-neutral measure (hereafter denoted by $\mathbb{Q}$ ), [1] assumes that the $\mathrm{N}$-dimensional state (factor) vector $Y$ follow the process

$$
Y_{t+1}=\mu_{t}^{Q^{j}}+\Sigma^{j} \epsilon_{t+1}
$$

where $\mu_{t}^{Q^{j}}=Y_{t}+\kappa^{Q}\left(\theta^{Q^{j}}-Y_{t}\right), \Sigma^{j}$ is a volatility matrix that is regime-dependent but not dependent on time, and $\epsilon_{t+1} \sim N\left(0, I_{N}\right)$ is standard normal.

The regime-switching $\mathbb{Q}$ probabilities $\pi^{Q}$ is state-independent. $\pi^{Q^{j k}}$ is the $(j, k)$ element of $\pi^{Q}$, denoting the $\mathbb{Q}$ probability of switching from regime $S_{t}=j$ to regime $S_{t+1}=k$.

The continuously compounded yield on a one-period zero-coupon bond in regime $j$ is assumed to be the affine function of $Y_{t}$, that is, $r_{t}^{j}=r\left(Y_{t}, S_{t}=j\right)=\delta_{0}^{j}+\delta_{Y} Y_{t}$.

Letting $D_{t, n}=D_{n}\left(Y_{t}, S_{t}\right)$ denote the time $t$ price for a zero-coupon bond with maturity of $n$ periods, and $D_{t, n}^{j}$ denote the price when the current regime is $S_{t}=j$. Then, as is proved by [1], we have,

$$
D_{t, n}^{j}=\exp \left(-A_{n}^{j}-B_{n} Y_{t}\right)
$$

where,

$$
\begin{gathered}
A_{n+1}^{j}=\delta_{0}^{j}+\left(\kappa^{Q} \theta^{Q^{j}}\right)^{\prime} B_{n}-\frac{1}{2} B_{n}^{\prime} \Sigma^{j} \Sigma^{j^{\prime}} B_{n}-\log \left(\sum_{k=0}^{s} \pi^{Q^{j k}} \mathrm{e}^{-A_{n}^{k}}\right) \\
B_{n+1}=\delta_{Y}+B_{n}-\kappa^{Q^{\prime}} B_{n}
\end{gathered}
$$

with initial conditions: $A_{0}^{j}=0, B_{0}=0$. When $n$ denotes maturities in months, the annualized yields are given by

$$
R_{t, n}^{j}=-\log \left(D_{t, n}^{j}\right) \times 12 / n=a_{n}^{j}+b_{n}^{\prime} Y_{t}
$$

where $a_{n}^{j}=\frac{A_{n}^{j}}{n / 12}, b_{n}=\frac{B_{n}}{n / 12}$.

The market prices of factor (MPF) risks in regime $j, \Lambda_{t}^{j}$, take a form of [5]'s essentially affine, assuming that

$$
\Lambda_{t}^{j}=\left(\Sigma^{j}\right)^{-1}\left(\lambda_{0}^{j}+\lambda_{Y}^{j} Y_{t}\right)
$$

Given the time $t+1$ regime $S_{t+1}=j$, under the historical measure (hereafter denoted by $P$ ), the N-dimensional state (factor) vector $Y$ follows the process

$$
Y_{t+1}=\mu_{t}^{P^{j}}+\Sigma^{j} \epsilon_{t+1}
$$

where $\mu_{t}^{P^{j}}=Y_{t}+\kappa^{P^{j}}\left(\theta^{P^{j}}-Y_{t}\right), \Sigma^{j}$ is a volatility matrix that is regime-dependent but not dependent on time, and $\epsilon_{t+1} \sim N\left(0, I_{N}\right)$ is standard normal.

The regime-switching $P$ probabilities $\pi_{t}^{P}$ is state-dependent. $\pi_{t}^{P^{j k}}$ is the $(j, k)$ element of $\pi_{t}^{P}$, denoting the $P$ probability of switching from regime $S_{t}=j$ to regime $S_{t+1}=k$. For the two-regime case, they assume,

$$
\pi_{t}^{P^{j k}}=\pi^{P^{j k}}\left(Y_{t}\right)=\frac{1}{1+\mathrm{e}^{\eta_{0}^{j k}+\eta_{Y}^{j k} Y_{t}}}, \quad \pi_{t}^{P^{j j}}=1-\pi_{t}^{P^{j k}}
$$

where $j \neq k$. And then, the market price of regime-shift (MPRS) risk from $S_{t}$ to $S_{t+1}$ is as, $\Gamma_{t}^{j k}=\log \left(\pi_{t}^{P^{j k}} / \pi_{t}^{Q^{j k}}\right), \forall j, k$.

\section{Identification of [1]'s Model}

[1] assumes that the yields on a collection of $N$ zero-coupon bonds are priced without error, and the yields on a collection of $M$ zero-coupon bonds are priced with error. Let $\hat{R}_{t}$ be the $N \times 1$ vector of yields for the bonds priced exactly by the model and $\tilde{R}_{t}$ be the remaining $M \times 1$ vector of yields for the bonds priced with 
error.

[1] belongs to the class of state space models. Any regime-switching Gaussian affine term structure model in which exactly $N$ yields are assumed to be priced without error takes the form of a restricted regime-switching multivariate linear regression (LR). The mapping from the affine-pricing parameters to the LR parameters allows us to evaluate the identifiability of a given structure. If two different values for the structural parameters imply the identical reduced-form parameters, there is no way to use observable data to choose between the two. Based on this idea, [8] demonstrates that [4] [11] and [12] are in fact unidentified. We now explore the implications of this fact for [1] described in the previous section.

Given the time $t$ regime $S_{t}=j$, according to (1), we have

$$
\hat{R}_{t}=\hat{a}^{j}+\hat{b} Y_{t}^{j}
$$

where $\hat{a}_{j}$ is a regime-dependent $N \times 1$ vector, $\hat{b}$ is a regime-independent $N \times N$ factor loading matrix. Inverting (2) results in

$$
Y_{t}^{j}=\hat{b}^{-1}\left(\hat{R}_{t}-\hat{a}^{j}\right)
$$

Then,

$$
\hat{R}_{t}=\hat{a}^{j}+\hat{b} Y_{t}^{j}=\hat{R}_{t-1}+\hat{K}_{0}^{j}+\hat{K}_{1}^{j} \hat{R}_{t-1}+\hat{\Sigma}^{j} \epsilon_{t}
$$

where,

$$
\begin{gathered}
\hat{K}_{0}^{j}=\hat{b} \kappa^{P^{j}} \theta^{P^{j}}+\hat{b} \kappa^{P^{j}} \hat{b}^{-1} \hat{a}^{j} \\
\hat{K}_{1}^{j}=-\hat{b} \kappa^{P^{j}} \hat{b}^{-1} \\
\hat{\Sigma}^{j}=\hat{b} \Sigma^{j} \\
\epsilon_{t} \sim N\left(0, I_{N}\right)
\end{gathered}
$$

The remaining $M$ yields can be expressed as follows,

$$
\tilde{R}_{t}=\tilde{a}^{j}+\tilde{b} Y_{t}^{j}+\Omega^{j} u_{t}=\tilde{K}_{0}^{j}+\tilde{K}_{1} \hat{R}_{t}+\Omega^{j} u_{t}
$$

where,

$$
\begin{gathered}
\tilde{K}_{0}^{j}=\tilde{a}^{j}-\tilde{b} \hat{b}^{-1} \hat{a}^{j} \\
\tilde{K}_{1}=\tilde{b} \hat{b}^{-1} \\
u_{t} \sim N\left(0, I_{M}\right)
\end{gathered}
$$

The $P$-measure regime-switching probability $\pi_{t}^{P^{j k}}$ can be transformed as follows,

$$
\pi_{t}^{p^{j k}}=\frac{1}{1+\mathrm{e}^{\eta_{0}^{j k}+\eta_{Y}^{j k} Y_{t}}}=\frac{1}{1+\mathrm{e}^{\hat{\eta}_{0}^{j k}+\eta_{R}^{j k} \hat{R}_{t}}}
$$

where,

$$
\hat{\eta}_{0}^{j k}=\eta_{0}^{j k}-\eta_{Y}^{j k} \hat{b}^{-1} \hat{a}^{j} ; \hat{\eta}_{R}^{j k}=\eta_{Y}^{j k} \hat{b}^{-1}
$$

Letting $\Theta_{\mathrm{ATSM}}=\left\{\delta_{0}^{j}, \delta_{Y}, \kappa^{P^{j}}, \theta^{P^{j}}, \Sigma^{j}, \lambda_{0}^{j}, \lambda_{Y}^{j}, \Omega^{j}, \pi^{Q}, \eta_{0}^{j k}, \eta_{Y}^{j k}\right\}$ be the vector of parameters relevant for regime-switching affine pricing and $\Theta_{\mathrm{LR}}=\left\{\hat{K}_{0}^{j}, \hat{K}_{1}^{j}, \tilde{K}_{0}^{j}, \tilde{K}_{1}^{j}, \hat{\Sigma}^{j}, \Omega^{j}, \hat{\eta}_{0}^{j k}, \hat{\eta}_{R}^{j k}\right\}$ be the vector of parameters in the regime-switching multivariate linear regression model. $\Theta_{\mathrm{LR}}$ is an implicit function of $\Theta_{\text {ATSM }}$. We know that $\Theta_{\text {ATSM }}$ have one-to-one correspondence to observations and thus are identifiable. Therefore, we examine whether the mapping from $\Theta_{\text {ATSM }}$ to $\Theta_{\mathrm{LR}}$ is one-to-one or not to determine the identifiability of $\Theta_{\text {ATSM }}$. If it is a 
multi-to-one mapping, then $\Theta_{\text {ATSM }}$ is unidentified; if it is a one-to-one mapping, then $\Theta_{\text {ATsM }}$ is just-identified; and if a one-to-multi mapping, then $\Theta_{\text {ATSM }}$ is over identified.

However, which kind of mapping it may be is not inherent in the model but depends on the data structure used. For example, if the dimension of $\tilde{R}_{t}$ (that is $M$ ) increases (or decreases) by 1, then the number of parameters in $\tilde{K}_{0}^{j}$ of $\Theta_{\mathrm{LR}}$ will increase (or decrease) by 1 , and the number of parameters in $\tilde{K}_{1}$ will increase (or decrease) by $N$. On the other side, all subsets of $\Theta_{\text {ATsm }}$ but $\Omega^{j}$ remain the same size. Therefore, the number of $M$ is crucial for the identification of $\Theta_{\text {ATSM }}$.

[1] estimates a two-regime, three-factor $(N=3)$ model. The vector $\hat{R}_{t}$ includes the yields on bonds with maturities of 6, 24, and 120 months, and $M=1$ with $\tilde{R}_{t}$ chosen to be the yield on the 60-month bond. The two regimes are denoted $L$ and $H$, corresponding to "low" and "high" values of the diagonal entries of $\Sigma^{j}$.

Firstly, let us look at the flexibility of [1]'s empirical model. They set $\kappa^{P^{L}}, \lambda_{Y}^{L}$ and $\lambda_{Y}^{H}$ as free parameters in their model. Consequently, $\kappa^{Q}$ as well as $\kappa^{P^{H}}$ are derived parameters using equations $\kappa^{Q}=\kappa^{P^{L}}+\lambda_{Y}^{L}$ and $\kappa^{P^{H}}=\kappa^{P^{L}}+\lambda_{Y}^{L}-\lambda_{Y}^{H}$. Furthermore, they set $\kappa^{P^{L}}$ to a lower triangular matrix which has 6 free parameers, and $\lambda_{Y}^{L}$ to a form of $\left[\begin{array}{ccc}0 & \lambda_{Y 12}^{L} & \lambda_{Y 13}^{L} \\ 0 & 0 & \lambda_{Y 23}^{L} \\ \lambda_{Y 31}^{L} & 0 & \lambda_{Y 33}^{L}\end{array}\right]$ which has 5 free parameters. However, even though $\kappa^{Q}$ is unrestricted full matrix, $\kappa^{Q}$ only has 9 parameters which is less than the sum of the number of parameters contained in $\kappa^{P^{L}}$ and $\lambda_{Y}^{L}$. In this case, there would be more than one set of $\kappa^{p^{L}}$ and $\lambda_{Y}^{L}$ that fulfill the equation $\kappa^{Q}=\kappa^{P^{L}}+\lambda_{Y}^{L}$ for some real $\kappa^{Q}$, making parameters $\kappa^{P^{L}}$ and $\lambda_{Y}^{L}$ unidentified. On the other hand, if $\kappa^{P^{L}}$ is determined by fitting the $P$ distribution of $Y_{t}$, then $\kappa^{Q}$ only has as many free parameters as $\lambda_{Y}^{L}$, therefore making $\kappa^{Q}$ an over-identified matrix. In this case, the model would be not so flexible.

Secondly, let us look at the total number of parameters for both models. Table 1 lists the number of free parameters contained in $\Theta_{\text {ATSM }}$ which is 49 . Besides, they fix some subsets of $\Theta_{\text {ATSM }}$ a priori, that is, $\delta_{0}^{L}=0.004417 ; \quad \delta_{0}^{H}+\delta_{Y}^{\prime} \theta^{P^{H}}=0.00767 ; \quad \theta^{P^{L}}=\left(\begin{array}{lll}0 & 0 & 0\end{array}\right)^{\prime}$ and $\sqrt{12} \Sigma^{L}=\left(\begin{array}{ccc}1 & & \\ & 1 & \\ & & 1\end{array}\right)$. These constraints reduce the total number of free parameters in $\Theta_{\text {ATsm }}$ to 41 . Table 2 lists the number of free parameters contained in $\Theta_{\mathrm{LR}}$ which is 34. Therefore, the only kind of mapping from $\Theta_{\mathrm{ATSM}}$ to $\Theta_{\mathrm{LR}}$ is multi-to-one, and there must be some parameters in $\Theta_{\text {ATSM }}$ which are unidentified.

\section{A New Representation and Its Estimation}

Due to the problems with identifiability of [1] parameters, we develop our "HW" canonical representation of regime-switching GDTSMs. Here, we use "HW" to represent [8], because they first propose this normalization for three-factor GDTSMs. However, they do not further examine this form of normalization.

In [8], they have proposed that for any $3 \times 3$ real-valued matrix:

$$
\rho=\left[\begin{array}{lll}
\rho_{11} & \rho_{12} & \rho_{13} \\
\rho_{21} & \rho_{22} & \rho_{23} \\
\rho_{31} & \rho_{32} & \rho_{33}
\end{array}\right]
$$

there exist $H$ that makes $\gamma=H \rho H^{-1}$, where $\gamma$ takes following form:

$$
\gamma=\left[\begin{array}{ccc}
\gamma_{11} & 0 & 0 \\
\gamma_{21} & a & \gamma_{23} \\
\gamma_{31} & \gamma_{32} & a
\end{array}\right]
$$

with $\gamma_{23} \leq \gamma_{32}$.

Although, as is pointed out in [8], this form cannot be extended to higher dimension, it has an advantage over others in that it can deal with the situation of $\kappa^{Q}$ having complex eigenvalues. This form is enough for us to 
Table 1. The number of free parameters in $\Theta_{\text {ATSM }}$.

\begin{tabular}{cccccc}
\hline $\begin{array}{c}\text { Coefficient } \\
\text { estimated }\end{array}$ & $\begin{array}{c}\text { Number of free } \\
\text { parameters }\end{array}$ & $\begin{array}{c}\text { Coefficient } \\
\text { estimated }\end{array}$ & $\begin{array}{c}\text { Number of free } \\
\text { parameters }\end{array}$ & $\begin{array}{c}\text { Coefficient } \\
\text { estimated }\end{array}$ & $\begin{array}{c}\text { Number of free } \\
\text { parameters }\end{array}$ \\
\hline$\kappa^{p^{L}}$ & 6 & $\theta^{p^{L}}$ & 3 & $\theta^{p^{H}}$ & 3 \\
$\lambda_{Y}^{L}$ & 5 & $\lambda_{Y}^{H}$ & 5 & $\lambda_{0}^{L}$ & 3 \\
$\lambda_{0}^{H}$ & 3 & $\Sigma^{H}$ & 3 & $\Sigma^{L}$ & 3 \\
$\delta_{Y}$ & 3 & $\delta_{0}^{L}$ & 1 & $\delta_{0}^{H}$ & 1 \\
$\pi^{Q^{L H}}$ & 1 & $\pi^{Q^{H L}}$ & 1 & $\eta_{0}^{L H}$ & 2 \\
$\eta_{0}^{H L}$ & 1 & $\eta_{Y}^{L H}$ & 2 & $\eta_{Y}^{H L}$ & \\
$\Omega^{L}$ & 1 & $\Omega^{H}$ & 1 & & \\
\hline
\end{tabular}

Table 2. The number of free parameters in $\Theta_{\mathrm{LR}}$.

\begin{tabular}{cccccc}
\hline $\begin{array}{c}\text { Coefficient } \\
\text { estimated }\end{array}$ & $\begin{array}{c}\text { Number of free } \\
\text { parameters }\end{array}$ & $\begin{array}{c}\text { Coefficient } \\
\text { estimated }\end{array}$ & $\begin{array}{c}\text { Number of free } \\
\text { parameters }\end{array}$ & $\begin{array}{c}\text { Coefficient } \\
\text { estimated }\end{array}$ & $\begin{array}{c}\text { Number of free } \\
\text { parameters }\end{array}$ \\
\hline$\hat{K}_{0}^{L}$ & 3 & $\hat{\Sigma}^{H}$ & 3 & $\tilde{K}_{0}^{H}$ & 1 \\
$\hat{K}_{0}^{H}$ & 3 & $\hat{\Sigma}^{L}$ & 3 & $\tilde{K}_{1}$ & 3 \\
$\hat{K}_{1}^{L}$ & 9 & $\tilde{K}_{0}^{L}$ & 1 & $\tilde{\Omega}^{H}$ & 1 \\
$\hat{K}_{1}^{H}$ & 9 & & & $\tilde{\Omega}^{L}$ & 1 \\
\hline
\end{tabular}

study regime-switching three-factor GDTSMs. Next, we propose an alternative normalization in the following Theorem.

Theorem 1. Every three-factor canonical GDTSM is observationally equivalent to the three-factor canonical GDTSM with

$$
\begin{gathered}
\Delta X_{t}=\gamma^{Q} X_{t-1}+\Sigma_{x} \epsilon_{t} \\
\Delta X_{t}=\gamma_{0}^{P}+\gamma_{1}^{P} X_{t-1}+\Sigma_{x} \epsilon_{t} \\
r_{t}=r_{\infty}+l^{\prime} X_{t}
\end{gathered}
$$

where, $\gamma^{Q}$ takes the following form:

$$
\gamma^{Q}=\left[\begin{array}{ccc}
\gamma_{11}^{Q} & 0 & 0 \\
\gamma_{21}^{Q} & a & \gamma_{23}^{Q} \\
\gamma_{31}^{Q} & \gamma_{32}^{Q} & a
\end{array}\right]
$$

$\gamma_{0}^{P}$ is a $3 \times 1$ vector, $\gamma_{1}^{P}$ is a $3 \times 3$ factor loading matrix, $l$ represents $\left(\begin{array}{lll}1 & 1 & 1\end{array}\right)^{\prime}, r_{\infty}$ is a scalar.

Proof:

Assuming some three-factor canonical GDTSM takes the following form:

$$
\begin{aligned}
& \Delta Y_{t}=\kappa_{0}^{Q}+\kappa_{1}^{Q} Y_{t-1}+\Sigma_{Y} \epsilon_{t}, \\
& \Delta Y_{t}=\kappa_{0}^{P}+\kappa_{1}^{P} Y_{t-1}+\Sigma_{Y} \epsilon_{t}, \\
& r_{t}=\delta_{0}+\delta_{Y} Y_{t} .
\end{aligned}
$$

For ease of exposition, we assume we have found $H$, making $H \gamma H^{-1}=\kappa_{1}^{Q}$, and $\gamma$ takes the form:

$$
\gamma=\left[\begin{array}{ccc}
\gamma_{11} & 0 & 0 \\
\gamma_{21} & a & \gamma_{23} \\
\gamma_{31} & \gamma_{32} & a
\end{array}\right]
$$

Then, letting $X_{t}=v^{-1}\left(Y_{t}+\left(\kappa_{1}^{Q}\right)^{-1} \kappa_{0}^{Q}\right)$, where $v=H \times \operatorname{diag}\left(\left(\delta_{Y}^{\prime} H\right)^{-1}\right)$. We can regard $X_{t}$ as a new state 
factor, because the mapping from $Y_{t}$ to $X_{t}$ is one-to-one. The $\mathbb{Q}$ dynamic process of $X_{t}$ can be obtained as follows,

$$
\begin{aligned}
\Delta X_{t} & =v^{-1} \Delta Y_{t}=v^{-1}\left(\kappa_{0}^{Q}+\kappa_{1}^{Q} Y_{t-1}+\Sigma_{Y} \epsilon_{t}\right) \\
& =\operatorname{diag}\left(\delta_{Y}^{\prime} H\right) \times H^{\prime} \kappa_{1}^{Q} H \times \operatorname{diag}\left(\left(\delta_{Y}^{\prime} H\right)^{-1}\right) X_{t-1}+v^{-1} \Sigma_{Y} \epsilon_{t} \\
& =\gamma^{Q} X_{x-1}+v^{-1} \Sigma_{Y} \epsilon_{t}
\end{aligned}
$$

where, $\gamma^{Q}=\operatorname{diag}\left(\delta_{Y}^{\prime} H\right) \times \gamma \times \operatorname{diag}\left(\left(\delta_{Y}^{\prime} H\right)^{-1}\right)$ which also takes the same form as $\gamma$, that is,

$$
\gamma^{Q}=\left[\begin{array}{ccc}
\gamma_{11}^{Q} & 0 & 0 \\
\gamma_{21}^{Q} & a & \gamma_{23}^{Q} \\
\gamma_{31}^{Q} & \gamma_{32}^{Q} & a
\end{array}\right]
$$

Likewise, the $\mathbb{P}$ dynamic process of $X_{t}$ can be obtained as follows,

$$
\Delta X_{t}=v^{-1} \Delta Y_{t}=v^{-1}\left(\kappa_{0}^{P}+\kappa_{1}^{P} Y_{t-1}+\Sigma_{\mathrm{Y}} \epsilon_{t}\right)=\gamma_{0}^{P}+\gamma_{1}^{P} X_{t-1}+v^{-1} \Sigma_{Y} \epsilon_{t}
$$

where, $\gamma_{0}^{P}=v^{-1} \kappa_{0}^{P}-v^{-1} \kappa_{1}^{P}\left(\kappa_{1}^{Q}\right)^{-1} \kappa_{0}^{P}, \gamma_{1}^{P}=v^{-1} \kappa_{1}^{P} v$. Both $\gamma_{0}^{P}$ and $\gamma_{1}^{P}$ are unrestricted vector and matrix because we do not impose any restriction on either $\kappa_{0}^{P}$ or $\kappa_{1}^{P}$.

Finally, we can transform the short rate as an affine function of the new state variables as follows,

$$
r_{t}=\delta_{0}+\delta_{Y} Y_{t}=\delta_{0}+\delta_{Y}\left(v X_{t}-\left(\kappa_{1}^{Q}\right)^{-1} \kappa_{0}^{Q}\right)=r_{\infty}+l^{\prime} X_{t}
$$

where, $r_{\infty}=\delta_{0}-\delta_{Y}\left(\kappa_{1}^{Q}\right)^{-1} \kappa_{0}^{Q}$ which is a scalar.

By Theorem 1, we will establish the reparametrization of [1] regime-switching three-factor GDTSM as follows.

Given the time $t$ regime $S_{t}=j$, under $\mathbb{Q}$, we assume that the three-dimensional state vector $X_{t}$ follow the process,

$$
\Delta X_{t}=\gamma^{Q} X_{t-1}+\Sigma_{x}^{j} \epsilon_{t}
$$

where $\gamma^{Q}$ takes the form of $\left[\begin{array}{ccc}\gamma_{11}^{Q} & 0 & 0 \\ \gamma_{21}^{Q} & a & \gamma_{23}^{Q} \\ \gamma_{31}^{Q} & \gamma_{32}^{Q} & a\end{array}\right], \Sigma_{x}^{j}$ is a volatility matrix that is regime-dependent but not dependent on time, and $\epsilon_{t} \sim N\left(0, I_{3}\right)$ is standard normal. Unlike [1], we do not have to set the intercept term. In order to have closed-form solutions for zero-coupon bond prices, we still set $\gamma^{Q}$ to be state-independent.

Like [1], the regime-switching $\mathbb{Q}$ probabilities $\pi^{Q}$ is state-independent. $\pi^{Q^{j k}}$ is the $(j, k)$ element of $\pi^{Q}$, denoting the $\mathbb{Q}$ probability of switching from regime $S_{t}=j$ to regime $S_{t+1}=k$.

Unlike [1], the continuously compounded yield on a one-period zero-coupon bond in regime $j$ is assumed to be a different affine function of $X_{t}$, that is, $r_{t}^{j}=r\left(Y_{t}, S_{t}=j\right)=r_{\infty}^{j}+l^{\prime} X_{t}$.

Then, given the time $t$ regime $S_{t}=j$, the time-t price for a zero-coupon bond with maturity of $n$ periods $D_{t, n}^{j}$ is computed as follows,

$$
D_{t, n}^{j}=\exp \left(-A_{n}^{j}-B_{n} X_{t}\right)
$$

where,

$$
\begin{gathered}
A_{n+1}^{j}=r_{\infty}^{j}-\frac{1}{2} B_{n}^{\prime} \Sigma_{X}^{j} \Sigma_{X}^{j{ }^{\prime}} B_{n}-\log \left(\sum_{k=0}^{s} \pi^{Q^{j k}} \mathrm{e}^{-A_{n}^{k}}\right) \\
B_{n+1}=l+B_{n}-\gamma^{Q} B_{n} .
\end{gathered}
$$

with initial conditions: $A_{0}^{j}=0, B_{0}=0$. When $n$ denotes maturities in months, the annualized yields are given 
by

$$
R_{t, n}^{j}=-\frac{\log \left(D_{t, n}^{j}\right)}{n \times \frac{1}{12}}=a_{n}^{j}+b_{n}^{\prime} X_{t}
$$

where $a_{n}^{j}=\frac{A_{n}^{j}}{n / 12}, b_{n}=\frac{B_{n}}{n / 12}$.

Given the time $t$ regime $S_{t}=j$, under $\mathbb{P}, X_{t}$ follows the process

$$
\Delta X_{t}=\gamma_{0}^{P^{j}}+\gamma_{1}^{p^{j}} X_{t-1}+\Sigma_{x}^{j} \epsilon_{t}
$$

where $\gamma_{0}^{P^{j}}, \gamma_{1}^{P^{j}}$ and $\Sigma_{x}^{j}$ are state-dependent parameters.

Like [1], the regime-switching $\mathbb{P}$ probabilities $\pi_{t}^{P}$ is state-dependent. For the two-regime case, we still assume,

$$
\pi_{t}^{p^{j k}}=\pi^{p^{j k}}\left(X_{t}\right)=\frac{1}{1+\mathrm{e}^{j_{0}^{j k}+\eta_{Y}^{j k} X_{t}}}, \pi_{t}^{p^{j j}}=1-\pi_{t}^{p^{j k}}, j=L / H, k \neq L
$$

And then, the market price of regime-shift (MPRS) risk from $S_{t-1}$ to $S_{t}$ is as, $\Gamma_{t}^{j k}=\log \left(\frac{\pi_{t}^{P^{j k}}}{\pi_{t}^{Q^{j k}}}\right), \forall j, k$.

Like [1], we could set the market prices of factor risks in regime $j, \Lambda_{t}^{j}=\left(\Sigma^{j}\right)^{-1}\left(\lambda_{0}^{j}+\lambda_{Y}^{j} Y_{t}\right)$. However, we do not set $\lambda_{0}^{j}$ and $\lambda_{x}^{j}$ to be free parameters. Instead, we set $\gamma^{Q}, \gamma_{0}^{p^{j}}$ and $\gamma_{1}^{P^{j}}$ as free parameters in our model. Consequently, $\lambda_{0}^{j}$ as well as $\lambda_{x}^{j}$ are derived parameters using equations $\lambda_{x}^{j}=\gamma^{Q}-\gamma_{1}^{P^{j}}$ and $\lambda_{0}^{j}=-\gamma_{0}^{P^{j}}$. A distinctive feature of this reparametrization is that, in estimation, there is an inherent separation between the parameters of the $\mathbb{P}$ and $\mathbb{Q}$ distributions of $X_{t}$. In contrast, when the risk factors are latent, estimates of the parameters governing the $\mathbb{P}$ distribution necessarily depend on those of the $\mathbb{Q}$ distribution of the state, since the pricing model is required to invert the model for the fitted states (when $N$ bonds are priced perfectly). We will formalize this "separation property" of our reparametrization in the following contents.

As in [1], we assume that the yields on a collection of three zero-coupon bonds are priced without error, and the yields on a collection of $M$ zero-coupon bonds are priced with error. In this data structure, we will prove that, for two-regime model, the sufficient condition of just-identification of our normalization is $M=2$.

Let $\hat{R}_{t}$ be the $3 \times 1$ vector of yields for the bonds priced exactly by the model and $\tilde{R}_{t}$ be the remaining $2 \times 1$ vector of yields for the bonds priced with error.

Given the time $t$ regime $S_{t}=j$, according to (1), we have

$$
\hat{R}_{t}=\hat{a}^{j}+\hat{b} X_{t}^{j}
$$

where $\hat{a}^{j}$ is a regime-dependent $3 \times 1$ vector, $\hat{b}$ is a regime-independent $3 \times 3$ factor loading matrix.

Inverting (2) results in

$$
X_{t}^{j}=\hat{b}^{-1}\left(\hat{R}_{t}-\hat{a}^{j}\right)
$$

Then,

$$
\hat{R}_{t}=\hat{a}^{j}+\hat{b} X_{t}^{j}=\hat{R}_{t-1}+\hat{K}_{0}^{j}+\hat{K}_{1}^{j} \hat{R}_{t-1}+\hat{\Sigma}^{j} \epsilon_{t}
$$

where,

$$
\begin{gathered}
\hat{K}_{0}^{j}=\hat{b} \gamma_{0}^{P^{j}}+\hat{b} \gamma_{1}^{P^{j}} \hat{b}^{-1} \hat{a}^{j}, \\
\hat{K}_{1}^{j}=-\hat{b} \gamma_{1}^{P^{j}} \hat{b}^{-1}, \\
\hat{\Sigma}^{j}=\hat{b} \Sigma_{X}^{j}, \\
\epsilon_{t} \sim N\left(0, I_{N}\right) .
\end{gathered}
$$


The remaining 2 yields can be expressed as follows,

$$
\tilde{R}_{t}=\tilde{a}^{j}+\tilde{b} X_{t}^{j}+\Omega^{j} u_{t}=\tilde{K}_{0}^{j}+\tilde{K}_{1} \hat{R}_{t}+\Omega^{j} u_{t}
$$

where,

$$
\begin{gathered}
\tilde{K}_{0}^{j}=\tilde{a}^{j}-\tilde{b} \hat{b}^{-1} \hat{a}^{j}, \\
\tilde{K}_{1}=\tilde{b} \hat{b}^{-1}, \\
u_{t} \sim N\left(0, I_{M}\right) .
\end{gathered}
$$

The $\mathbb{P}$-measure regime-switching probability $\pi_{t}^{p^{j k}}$ can be transformed as follows,

$$
\pi_{t}^{p^{j k}}=\frac{1}{1+\mathrm{e}^{\eta_{0}^{j k}+\eta_{X}^{j k} X_{t}}}=\frac{1}{1+\mathrm{e}^{\hat{\eta}_{0}^{j k}+\hat{\eta}_{R}^{j k} \hat{R}_{t}}}
$$

where,

$$
\begin{gathered}
\hat{\eta}_{0}^{j k}=\eta_{0}^{j k}-\eta_{X}^{j k} \hat{b}^{-1} \hat{a}^{j} \\
\hat{\eta}_{R}^{j k}=\eta_{X}^{j k} \hat{b}^{-1}
\end{gathered}
$$

In summary, we can use the method proposed in [13] to estimate the parameters $\Theta_{\mathrm{LR}}=\left\{\hat{K}_{0}^{j}, \hat{K}_{1}^{j}, \tilde{K}_{0}^{j}, \tilde{K}_{1}, \hat{\Sigma}^{j}, \Omega^{j}, \hat{\eta}_{0}^{j k}, \hat{\eta}_{R}^{j k}\right\}$, then we can back out our state-space parameters $\Theta_{\text {ATSM }}=\left\{r_{\infty}^{j}, \gamma^{Q}, \gamma_{0}^{p^{j}}, \gamma_{1}^{p^{j}}, \Sigma_{X}^{j}, \pi^{Q^{j k}}, \eta_{0}^{j k}, \eta_{X}^{j k}\right\}$ as follows.

Step 1. The estimate of the 6 unknowns in $\gamma^{Q}$ is obtained by numerically solving the 6 equations in $\tilde{K}_{1}=\tilde{b} \hat{b}^{-1}$.

Step 2. The estimate of $\Sigma_{X}^{j}$ is obtained analytically by the equation $\hat{\Sigma}^{j}=\hat{b} \Sigma_{X}^{j}$, that is $\Sigma_{X}^{j}=\hat{b}^{-1} \hat{\Sigma}^{j}$.

Step 3. The estimate of the 4 unknowns in $\left\{r_{\infty}^{L}, r_{\infty}^{H}, \pi^{Q^{L H}}, \pi^{Q^{H L}}\right\}$ is obtained by numerically solving the 4 equations in

$$
\tilde{K}_{0}^{L}=\tilde{a}^{L}-\tilde{b} \hat{b}^{-1} \hat{a}^{L}=\tilde{a}^{L}-\tilde{K}_{1} \hat{a}^{L}
$$

and,

$$
\tilde{K}_{0}^{H}=\tilde{a}^{H}-\tilde{K}_{1} \hat{a}^{H}
$$

Step 4. The estimate of $\gamma_{1}^{P^{j}}$ is obtained analytically by the equation $\hat{K}_{1}^{j}=-\hat{b} \gamma_{1}^{p^{j}} \hat{b}^{-1}$, that is $\gamma_{1}^{p^{j}}=-\hat{b}^{-1} \hat{K}_{1}^{j} \hat{b}$.

Step 5. The estimate of $\gamma_{0}^{P^{j}}$ is obtained analytically by the equation $\hat{K}_{0}^{j}=\hat{b} \gamma_{0}^{P^{j}}+\hat{b} \gamma_{1}^{p^{j}} \hat{b}^{-1} \hat{a}^{j}=\hat{b} \gamma_{0}^{P^{j}}-\hat{K}_{1}^{j} \hat{a}^{j}$, that is $\gamma_{0}^{p^{j}}=\hat{b}^{-1}\left(\hat{K}_{0}^{j}+\hat{K}_{1}^{j} \hat{a}^{j}\right)$.

Step 6. The estimate of $\eta_{X}^{j k}$ is obtained analytically by the equation $\hat{\eta}_{R}^{j k}=\eta_{X}^{j k} \hat{b}^{-1}$, that is $\eta_{X}^{j k}=\eta_{R}^{j k} \hat{b}$.

Step7. The estimate of $\eta_{0}^{j k}$ is obtained analytically by the equation $\hat{\eta}_{0}^{j k}=\eta_{0}^{j k}-\eta_{X}^{j k} \hat{b}^{-1} \hat{a}^{j}=\eta_{0}^{j k}-\hat{\eta}_{R}^{j k} \hat{a}^{j}$ that is $\eta_{0}^{j k}=\hat{\eta}_{0}^{j k}+\hat{\eta}_{R}^{j k} \hat{a}^{j}$.

In every step, the solving processes can be invertible, so we can also obtain $\Theta_{\mathrm{LR}}$ from $\Theta_{\mathrm{ATSM}}$. That is the mapping relation between $\Theta_{\mathrm{LR}}$ and $\Theta_{\mathrm{ATSM}}$ is one-to-one, and the parameters of our normalization are justidentified.

When $M=1$, the situation is different. In Step 1, there are still 6 unknowns in $\gamma^{Q}$, while there are only 3 equations in $\tilde{K}_{1}=\tilde{b} \hat{b}^{-1}$; in Step 3, we still need estimate the 4 unknowns in $\left\{r_{\infty}^{L}, r_{\infty}^{H}, \pi^{Q^{L H}}, \pi^{Q^{H L}}\right\}$, while there are only 2 equations in $\tilde{K}_{0}^{L}=\tilde{a}^{L}-\tilde{K}_{1} \hat{a}^{L}$ and $\tilde{K}_{0}^{H}=\tilde{a}^{H}-\tilde{K}_{1} \hat{a}^{H}$. The mapping from $\Theta_{\mathrm{ATSM}}$ to $\Theta_{\mathrm{LR}}$ is mul- 
ti-to-one and so the parameters of our normalization are unidentified.

When $M \geq 3$, we will see our state-space parameters $\Theta_{\text {ATsM }}$ to be over identified. In Step 1, there will be at least $3 \times 3=9$ equations, but we still need to estimate only 6 unknowns in $\gamma^{Q}$. In Step 3, there will be at least 3 $\times 2=6$ equations in $\tilde{K}_{0}^{L}=\tilde{a}^{L}-\tilde{K}_{1} \hat{a}^{L}$ and $\tilde{K}_{0}^{H}=\tilde{a}^{H}-\tilde{K}_{1} \hat{a}^{H}$, but we there are still only 4 unknowns in $\left\{r_{\infty}^{L}, r_{\infty}^{H}, \pi^{Q^{L H}}, \pi^{Q^{H L}}\right\}$. This means that the mapping from $\Theta_{\text {ATSM }}$ to $\Theta_{\mathrm{LR}}$ is one-to-multi and so the parameters of our normalization are over identified.

The next question is how to obtain the standard error for these state-space parameters $\Theta_{\text {ATsM }}$. [8] has proved that under the usual regularity conditions, we could use Delta Methods to obtain the asymptotic standard errors of the structural parameters.

Within [1]'s parametrization, the $\mathbb{Q}$ parameters control the cross-sectional relationship among the yields and the latent factors are fitted from observed yields, so the estimates of the parameters governing the $\mathbb{P}$ distribution will necessarily depend on those of the $\mathbb{Q}$ distribution of the state. On the other hand, [1]'s parametrization also makes the $\mathbb{Q}$ parameters as derived parameters from $\mathbb{P}$ parameters. Therefore, we cannot back out $\mathbb{Q}$ parameters and $\mathbb{P}$ parameters separately in [1]'s model, while our model's parametrization makes this "separation property" possible.

\section{Regime-Switching Three-Factor Arbitrage-Free Nelson-Siegel Model.}

In this section, we will show that the regime-switching extension on the AFNS model of [9] is a constrained special case of our representation.

By [9], under $\mathbb{Q}$, the three-dimensional state vector $Z_{t}$ follow the process,

$$
\left(\begin{array}{l}
d Z_{t}^{1} \\
d Z_{t}^{2} \\
d Z_{t}^{3}
\end{array}\right)=\left(\begin{array}{ccc}
0 & 0 & 0 \\
0 & \lambda & -\lambda \\
0 & 0 & \lambda
\end{array}\right)\left[\left(\begin{array}{c}
\theta_{1}^{Q} \\
\theta_{2}^{Q} \\
\theta_{3}^{Q}
\end{array}\right)-\left(\begin{array}{c}
Z_{t}^{1} \\
Z_{t}^{2} \\
Z_{t}^{3}
\end{array}\right)\right]+\Sigma_{z} \omega_{t}
$$

where $\Sigma_{Z}$ is a volatility matrix and $\omega_{t} \sim N\left(0, I_{3}\right)$ is standard normal. The short rate depends only on the first two latent pricing factors, that is,

$$
r_{t}=Z_{t}^{1}+Z_{t}^{2}
$$

First, we let $F_{t}=Z_{t}-\theta^{Q}$ to be a new state vector. Replacing $Z_{t}$ with $F_{t}$ in (6) and (7), we have,

$$
\begin{aligned}
\left(\begin{array}{l}
d F_{t}^{1} \\
d F_{t}^{2} \\
d F_{t}^{3}
\end{array}\right)=\left(\begin{array}{ccc}
0 & 0 & 0 \\
0 & -\lambda & \lambda \\
0 & 0 & -\lambda
\end{array}\right)\left(\begin{array}{l}
F_{t}^{1} \\
F_{t}^{2} \\
F_{t}^{3}
\end{array}\right)+\Sigma_{z} \omega_{t} \\
r_{t}=r_{\infty}+\left(\begin{array}{lll}
1 & 1 & 0
\end{array}\right)\left(\begin{array}{l}
F_{t}^{1} \\
F_{t}^{2} \\
F_{t}^{3}
\end{array}\right)
\end{aligned}
$$

where

$$
r_{\infty}=\theta_{1}^{Q}+\theta_{2}^{Q} .
$$

Second, let $X_{t}=G F_{t}$, with $G=\left[\begin{array}{ccc}1 & 0 & 0 \\ 0 & 1 & -\lambda \\ 0 & 0 & \lambda\end{array}\right]$. Premultiply both sides of (8) by $G$, we have,

$$
\left(\begin{array}{l}
d X_{t}^{1} \\
d X_{t}^{2} \\
d X_{t}^{3}
\end{array}\right)=\left(\begin{array}{ccc}
0 & 0 & 0 \\
0 & -\lambda & \lambda \\
0 & 0 & -\lambda
\end{array}\right)\left(\begin{array}{c}
X_{t}^{1} \\
X_{t}^{2} \\
X_{t}^{3}
\end{array}\right)+\Sigma_{X} \omega_{t}
$$


where $\Sigma_{X}=G \Sigma_{Z}$. Inserting $F_{t}=G^{-1} X_{t}=\left[\begin{array}{ccc}1 & 0 & 0 \\ 0 & 1 & 1 \\ 0 & 0 & \frac{1}{\lambda}\end{array}\right] X_{t}$ into (7) produces

where $l=\left(\begin{array}{lll}1 & 1 & 1\end{array}\right)^{\prime}$.

Comparing (10) with (3), we find that the regime-switching AFNS model is the constrained special case of the our normalization with $\gamma_{11}^{Q}=0, \quad \gamma_{21}^{Q}=0, \quad \gamma_{31}^{Q}=0, \gamma_{32}^{Q}=0$ and $\gamma_{23}^{Q}=-a$.

Sometimes, we want to test if these constraints are valid. We could set regime-switching AFNS model as the null model and our representation as the alternative model, and then, under a desired statistical significance level, we compare likelihood ratio to the chi squared value with degrees of freedom equal to 5 .

\section{Conclusions}

[1]'s regime-switching three-factor affine term structure model, when we assume that the yields on a collection of three zero-coupon bonds are priced without error, is simply a restricted regime-switching linear regression. We use this correspondence to demonstrate that [1]'s parameters are in fact universally unidentified and a subset of their parameterization is over identified. As a solution to the problem with the identifiability, we propose a canonical representation of GDTSMs based on [8]'s proposal, which is then used in double-regime environment as a new form of regime-switching GDTSM. We also demonstrate that the parameters of our new form of regime-switching GDTSM are just-identified when the number of the pricing-with-error yields M equals 2. Our model's parametrization has another advantage over [1] in that we can back out $\mathbb{Q}$ parameters and $\mathbb{P}$ parameters separately and make the estimation of structural parameters easier. Finally, we show that regimeswitching three-factor arbitrage-free dynamic Nelson-Siegel model is a restricted special case of our model.

Besides, due to the tremendous numerical challenges in estimating the necessary parameters, we hope that our method will help to make these models a more effective tool for research in better describing the historical interest rate data.

\section{Acknowledgements}

This work is supported by Research Innovation Foundation of Shanghai University of Finance and Economics under Grant No. CXJJ-2013-321. And I am especially grateful to Professor Hong Li for his support and encouragement. All errors are my own.

\section{References}

[1] Dai, Q., Singleton, K.J. and Yang, W. (2007) Regime Shifts in a Dynamic Term Structure Model of US Treasury Bond Yields. Review of Financial Studies, 20, 1669-1706. http://dx.doi.org/10.1093/rfs/hhm021

[2] Vasicek, O. (1977) An Equilibrium Characterization of the Term Structure. Journal of Financial Economics, 5, 177188. http://dx.doi.org/10.1016/0304-405X(77)90016-2

[3] Duffie, D. and Kan, R. (1996) A Yield-Factor Model of Interest Rates. Mathematical Finance, 6, 379-406. http://dx.doi.org/10.1111/j.1467-9965.1996.tb00123.x

[4] Dai, Q. and Singleton, K. (2000) Specification Analysis of Affine Term Structure Models. Journal of Finance, 55, 1943-1978. http://dx.doi.org/10.1111/0022-1082.00278

[5] Duffee, G.R. (2002) Term Premia and Interest Rate Forecasts in Affine Models. Journal of Finance, 57, 405-443. http://dx.doi.org/10.1111/1540-6261.00426

[6] Garcia, R. and Perron, P. (1996) An Analysis of the Real Interest Rate Under Regime Shifts. The Review of Economics and Statistics, 78, 111-125. http://dx.doi.org/10.2307/2109851

[7] Ang, A. and Bekaert, G. (2002) Short Rate Nonlinearities and Regime Switches. Journal of Economic Dynamics and Control, 26, 1243-1274. http://dx.doi.org/10.1016/S0165-1889(01)00042-2

[8] Hamilton, J. and Wu, J. (2012) Identification and Estimation of Gaussian Affine Term Structure Models. Journal of 
Econometrics, 168, 315-331. http://dx.doi.org/10.1016/j.jeconom.2012.01.035

[9] Christensen, J.H.E., Diebold, F.X. and Rudebusch, G.D. (2011) An Arbitrage-Free Class of Nelson-Siegel Term Structuremodel. Journal of Econometrics, 164, 4-20. http://dx.doi.org/10.1016/j.jeconom.2011.02.011

[10] Bandara, W. and Munclinger, R. (2011) Term Structure Modeling with Structural Breaks: A Simple Arbitrage-Free Approach. http://ssrn.com/abstract=1974033 http://dx.doi.org/10.2139/ssrn.1974033

[11] Ang, A. and Piazzesi, M. (2003) A No-Argitrage Vector Autoregression of Term Structure Dynamics with Macroeconomic and Latent Variables. Journal of Monetary Economics, 50, 745-787. http://dx.doi.org/10.1016/S0304-3932(03)00032-1

[12] Pericoli, M. and Taboga, M. (2008) Canonical Term-Structure Models with Observable Factors and the Dynamics of Bond Risk Premia. Journal of Money, Credit and Banking, 40, 1471-1488. http://dx.doi.org/10.1111/j.1538-4616.2008.00167.x

[13] Hamilton, J. (1989) A New Approach to the Economic Analysis of Non-Stationary Time Series and the Business Cycle. Econometrica, 57, 357-384. http://dx.doi.org/10.2307/1912559 